\title{
Tradução e adaptação cultural da Barratt Impulsiveness Scale (BIS-11) para aplicação em adultos brasileiros
}

Translation and cultural adaptation of Barratt Impulsiveness Scale (BIS-11) for administration in Brazilian adults

Leandro Fernandes Malloy-Diniz',2, Paulo Mattos ${ }^{2,3}$, Wellington Borges Leite, Neander Abreu, ${ }^{2,4}$, Gabriel Coutinho $0^{2,3}$, Jonas Jardim de Paula1,2, Hermano Tavares ${ }^{5}$, Alina Gomide Vasconcelos ${ }^{1,2}$, Daniel Fuentes, ${ }^{2,5}$

\section{RESUMO}

Objetivos: Os objetivos deste estudo foram traduzir, adaptar culturalmente e verificar a equivalência literal, semântica e idiomática da Barratt Impulsiveness Scale (BIS-11), que avalia a presença de manifestações da impulsividade tendo como base o modelo teórico proposto por Ernst Barratt. Métodos: Inicialmente, a versão original em inglês da BIS-11 foi traduzida para o português por seis pesquisadores bilíngues. Em seguida, foi realizada uma tradução reversa para o inglês por uma tradutora de origem norte-americana. As versões original, traduzida e retraduzida foram avaliadas por um comitê de juízes especialistas, os quais emitiram pareceres com as observações pertinentes, o que culminou em uma versão final traduzida da BIS-11. As versões original e traduzida foram aplicadas em duas amostras da população

\section{Palavras-chave}

Impulsividade, psicometria, escalas, personalidade.

\section{Keywords}

Impulsivity, psychometry, scales, personality. geral com proficiência na língua inglesa, a fim de investigar a equivalência literal, semântica e idiomática da versão traduzida por meio de análises de correlação. Conclusão: Os resultados das análises quantitativas indicaram que a versão final do instrumento é satisfatória.

\section{ABSTRACT}

Objectives: The objective of this study was to translate, make transcultural adaptation and assess the semantic, idiomatic and literal equivalence of the Barratt Impulsiveness Scale (BIS-11). Methods: This scale assesses the presence of impulsive manifestations from the theoretical model proposed by Ernst Barratt. Firstly, the BIS-11 original version in English was translated to Portuguese by six bilingual researches. After this, was made the back-translation to English by a translator that was born in United States. Then, the three versions (original, translated and back-translated) were assessed by a specialists committee which made and analyze and comments about the process and then we reach the final translated version of BIS-11. The original and translated version of BIS-11 was applied in two samples from general population with proficiency both in English and Portuguese. This method was adopted to assess the literal, semantic e idiomatic equivalence of these versions by mean of correlation analyses. Conclusion: The final results of quantitative analyses show that the final version of BIS-11 is satisfactory.
1 Universidade Federal de Minas Gerais (UFMG), Programa de Pós-Graduação em Neurociências.

2 Laboratórios Integrados de Neuropsicologia (Lineu).

3 Universidade Federal do Rio de Janeiro (UFRJ), Instituto de Psiquiatria.

4 Universidade Federal da Bahia (UFBA), Instituto de Psicologia.

5 Universidade de São Paulo (USP), Instituto de Psiquiatria.

Endereço para correspondência: Leandro Fernandes Malloy-Diniz Faculdade de Filosofia e Ciências Humanas - Departamento de Psicologia Avenida Presidente Antônio Carlos, 6627, Pampulha - 31270-901 - Belo Horizonte, MG E-mail:malloy.diniz@gmail.com 


\section{INTRODUÇÃO}

A impulsividade é um fenótipo complexo caracterizado por diferentes padrões cognitivos e comportamentais que levam a consequências disfuncionais imediatas e em médio/ longo prazo. De acordo com Moeller et al.', a impulsividade ocorre quando: (1) há mudanças no curso da ação sem que seja feito um julgamento consciente prévio; (2) ocorrem comportamentos impensados; (3) se manifesta uma tendência a agir com menor nível de planejamento em comparação a indivíduos com mesmo nível intelectual.

Diversos são os transtornos em que as diferentes manifestações da impulsividade se apresentam de forma intensa, gerando prejuízos para a o indivíduo e aqueles que o cercam. Tanto nos transtornos do controle do impulso (jogo patológico, piromania, cleptomania, tricotilomania, oniomania) quanto em transtornos de personalidade (personalidade antissocial, personalidade borderline, personalidade explosivo intermitente) e outros descritos nos eixos I e II do DSM-IV (dependência e abuso de substâncias, transtorno afetivo bipolar, transtorno de conduta e o transtorno do déficit de atenção e hiperatividade) podem ocorrer manifestações de impulsividade ${ }^{1-6}$.

Mesmo em situações em que a impulsividade não está associada ao quadro sintomático de um transtorno, uma maior expressão desse traço fenotípico pode levar a prejuízos importantes. Por exemplo, há evidências da relação entre impulsividade e comportamento de risco no trânsito?.

O impacto e os prejuízos das diferentes manifestações da impulsividade em diversas situações do cotidiano, seja na presença de transtornos mentais ou não, justificam o investimento no desenvolvimento de medidas de avaliação, prevenção e tratamento. Tais medidas, entretanto, devem ter como base modelos teóricos consistentes e capazes de explicar as diferentes manifestações da impulsividade, inclusive em termos neurobiológicos.

Um dos modelos mais influentes na explicação do comportamento impulsivo foi proposto por Ernst Barratt. Na versão atual do modelo, a impulsividade é concebida como apresentando três componentes distintos, a saber: a) motor: a impulsividade motora está relacionada a não inibição de respostas incoerentes com o contexto; b) atencional: impulsividade relacionada à tomada de decisão rápida e c) falta de planejamento: engloba comportamentos orientados para o presente $^{8}$. A escala de autorrelato desenvolvida por Barratt para avaliar essas dimensões da impulsividade é denominada Barratt Impulsiveness Scale (BIS-11), e evidências de validade foram obtidas nos estudos desenvolvidos por Patton et al. ${ }^{8}$, em uma amostra composta por 412 universitários, 248 pacientes psiquiátricos e 73 presidiários do sexo masculino. Os resultados da análise fatorial exploratória pelo método dos componentes principais e rotação oblíqua identificaram seis fatores de primeira ordem e três fatores de segunda ordem, e dois deles são semelhantes ao modelo teórico. No entanto, o terceiro fator de segunda ordem não coincide com a definição conceitual de impulsividade cognitiva. Os índices de consistência interna foram altos e variaram entre 0,79 e 0,82, considerando cada uma das amostras. Por fim, a análise de variância univariada indicou que os grupos se diferenciam quanto às medidas de impulsividade $(F=27,49 ; p<0,001)$; os universitários apresentam escores mais baixos em relação aos pacientes psiquiátricos e aos presidiários.

Existem estudos com versões brasileiras da BIS em populações clínicas ${ }^{2-3}$ e em adolescentes do sexo masculino ${ }^{8}$. No estudo conduzido por Diemen et al. ${ }^{9}$, o índice de consistência interna da BIS11-A foi satisfatório $(0,62)$, no entanto não se obtiveram os três fatores teóricos originais propostos. No entanto, não se encontram na literatura estudos de adaptação transcultural da BIS-11 e avaliação de suas propriedades psicométricas quando aplicadas em adultos brasileiros. Assim, os objetivos do presente estudo consistiram em: a) descrever o processo de tradução e adaptação da Barratt Impulsiveness Scale (BIS-11) para o português no contexto brasileiro e b) avaliar a consistência entre as respostas dadas a BIS-11 em sua versão original e na versão traduzida por dois grupos de jovens adultos tidos como proficientes em sua língua materna, o português, e em inglês.

\section{MÉTODO}

\section{Procedimentos e participantes}

O estudo foi dividido em cinco etapas e todos os participantes consentiram em disponibilizar os dados obtidos para o estudo. Na primeira etapa, a versão original da BIS-11 foi traduzida conjuntamente por um grupo de especialistas. Participaram dessa etapa seis pesquisadores bilíngues com ampla experiência clínica e em pesquisa sobre avaliação e tratamento de transtornos relacionados à impulsividade. Os especialistas apresentavam diferentes formações profissionais (um psiquiatra, quatro psicólogos e um neurologista) e eram provenientes de quatro estados diferentes do Brasil (Minas Gerais, São Paulo, Rio de Janeiro e Bahia), o que contribuiu para que fossem evitados termos regionais. Todos os participantes dessa etapa apresentavam pós-graduação na área da saúde e quatro apresentavam título de doutor.

Na segunda etapa, uma tradutora juramentada de origem norte-americana, fluente em português e em inglês, fez a tradução reversa da versão obtida na primeira etapa. $\mathrm{Na}$ terceira etapa, o mesmo grupo de especialistas que participou da primeira etapa avaliou a equivalência literal, idiomática e semântica entre a tradução e a retrotradução. A partir do resultado dessa análise foi obtida uma versão nova da escala denominada versão síntese.

$\mathrm{Na}$ quarta etapa, as versões original e síntese do instrumento foram aplicadas em duas amostras bilíngues compostas por adultos recrutados a partir da rede social dos pesquisadores. A amostra bilíngue 1 foi composta por 25 (48\% 
do sexo feminino) participantes que apresentavam fluência comprovada por certificado de proficiência no idioma inglês. Todos apresentavam ensino superior ( $48 \%$ incompleto e 52\% completo) e idade média de $28,9(\mathrm{dp}=10,3)$ anos.

Na quinta etapa, uma segunda amostra bilíngue foi composta por 20 participantes que moravam, em média, há 7,05 $(\mathrm{dp}=2,14)$ anos nos Estados Unidos. Os participantes dessa amostra apresentavam idade média de 38,6 ( $\mathrm{dp}=9,40)$ anos, sendo $50 \%$ do sexo masculino. Em relação à escolaridade, $65 \%$ apresentaram superior completo/em formação, 25\%, pós-graduação e 10\%, segundo grau completo. Além disso, para aperfeiçoar a compreensão dos aspectos que interferiram na correlação entre os itens das duas versões, a amostra bilíngue 2 respondeu a um questionário sobre os três itens da versão final traduzida que não apresentaram boas propriedades psicométricas (itens 4, 20 e 26) nas análises da quarta etapa. Com esse intuito, foram-lhes apresentados critérios específicos desenvolvidos para esse estudo para a avaliação da equivalência das diferentes das versões, a saber: a) equivalência literal: verificar se a tradução corresponde literalmente à versão original; b) equivalência semântica: se há equivalência com relação ao sentido da palavra traduzida, a despeito da equivalência literal; c) equivalência idiomática: verificar se há correspondência no significado de expressões coloquiais e/ou gírias em dois contextos. Os avaliadores deveriam vincular sua avaliação a uma escala Likert cujas respostas poderiam variar de 0 a 2 , a saber: 0 = não equivalente; $1=$ indeciso; 2 = equivalente.

O projeto de pesquisa foi submetido e aprovado pelo Comitê de Ética em pesquisa da Universidade FUMEC. Todos os sujeitos envolvidos no estudo assinaram um termo de consentimento livre e esclarecido referente à sua participação na pesquisa.

\section{Instrumento}

A BIS-11 é uma escala de autopreenchimento composta por 30 itens relacionados às manifestações da impulsividade, tendo como base o modelo teórico proposto por Ernst Barratt ${ }^{8,10}$. O probando deve analisar cada um dos itens considerando seu próprio comportamento e classificá-los de acordo com uma escala do tipo Likert de quatro pontos, a saber: 1 = raramente ou nunca; 2 = de vez em quando; 3 = com frequência; 4 = quase sempre/sempre. A pontuação da escala varia de 30 a 120 pontos, e altos escores indicam a presença de comportamentos impulsivos. Além de um escore global, a BIS-11 permite o cálculo de escores parciais referentes a três subdomínios da impulsividade, sendo eles a impulsividade motora (itens $2,3,4,16,17,19,21,22,23,25$ e $30^{*}$ ), atencional (itens $6,5,9^{*}, 11,20^{*}, 24,26,28$ ) e por não planejamento (itens $\left.1^{*}, 7^{*}, 8^{*}, 10^{*}, 12^{*}, 13^{*}, 14,15^{*}, 18,27,29^{*}\right)^{1}$.

1 Os itens marcados com o sinal * recebem escore inverso para o cálculo dos escores parciais e total $(4,3,2,1)$.

\section{Análise dos dados}

A análise dos dados pode ser dividida em três fases. Inicialmente, foram realizadas estatísticas descritivas para caracterizar aspectos sociodemográficos dos participantes. Nas etapas 1 a 3 do estudo, os resultados foram obtidos a partir da comparação qualitativa das três versões, a fim de julgar a pertinência da versão retrotraduzida dos itens em comparação às versões original e traduzida. Já na fase 4 , foi realizada avaliação da consistência entre as respostas dadas a BIS-11 em sua versão original e na versão final traduzida por dois grupos de adultos tidos como proficientes em língua portuguesa e em inglês. Para isso, foram calculados os coeficientes de correlação de Spearman. Supõe-se que, caso se tratassem de versões equivalentes, seriam obtidas correlações significativas de magnitude variando de moderada a alta, encontradas entre as respostas as duas versões do instrumento. No intuito de compreender a não obtenção de correlações não significativas, foi realizada análise da consistência das respostas por meio da análise de correlação item-total.

Finalmente, a avaliação realizada pela amostra bilíngue 2 , quanto à pertinência da tradução dos itens originalmente apresentados na língua inglesa para o português brasileiro, foi analisada separadamente para cada item, por meio de estatísticas descritivas.

As análises quantitativas foram realizadas a partir do uso do software SPSS (Statistical Package for Social Sciences), versão 15.0 .

\section{RESULTADOS}

a) Análises qualitativas da tradução e retrotradução dos itens: Os resultados referente às etapas 1 a 3 consistiram em dados qualitativos obtidos a partir da comparação das versões da tradução direta e reversa da BIS-11 pelos especialistas (Quadro 1). Dos 30 itens que compõem a escala, identificouse a necessidade de aperfeiçoamento da tradução de três itens, a saber: itens 1, 23 e 30. As versões traduzidas do item 1 ("I plan tasks carefully"), do item 23 ("I can only think about one thing at a time") e do item 30 ("I am future oriented") foram consideradas inadequadas idiomaticamente. Isso levou à modificação da primeira versão traduzida do item 1 de "Eu planejo as coisas cuidadosamente" para "Eu planejo tarefas cuidadosamente"; do item 23 de "Eu só consigo pensar em um problema de cada vez" para "Eu só consigo pensar em uma coisa de cada vez"; e, finalmente, da tradução inicial do item 30 de "Eu me planejo para o futuro" para "Eu me preparo para o futuro". Tais modificações tiveram como intuito oferecer maior proximidade idiomática com o termo presente na versão original do instrumento. Por fim, foi elaborada a versão síntese, considerada versão final da adaptação da BIS-11 para o idioma português praticado no Brasil, composta por 30 itens, conforme apresentado na coluna 4 do Quadro 1. 
Quadro 1. Procedimentos do processo de tradução do instrumento BIS-11

\begin{tabular}{ccccc}
\hline \multicolumn{3}{c}{ Itens } & & Síntese da análise comparativa da tradução e retrotradução \\
Versão original & $\begin{array}{c}\text { Versão traduzida } \\
\text { (etapa 1) }\end{array}$ & $\begin{array}{c}\text { Versão retrotradução } \\
\text { (etapa 2) }\end{array}$ & $\begin{array}{c}\text { Versão final } \\
\text { (etapa 3) }\end{array}$ & \begin{tabular}{c} 
(etapa 3) \\
\hline
\end{tabular} \\
\hline
\end{tabular}

$1 \quad$ I plan tasks carefully. Eu planejo as coisas I plan things carefully. Eu planejo tarefas
cuidadosamente. cuidadosamente.

0 uso do termo "coisas" apresenta sentido semelhante ao de tarefas. No entanto, na versão final, optou-se pelo termo "tarefas", pelo fato de ele apresentar maior proximidade literal, semântica e idiomática com o termo original.

$\begin{array}{lllll}23 & \begin{array}{l}\text { Ido things without } \\ \text { thinking. }\end{array} & \begin{array}{l}\text { Eu faço coisas sem } \\ \text { pensar. }\end{array} & \begin{array}{l}\text { Ido things without } \\ \text { thinking. }\end{array} & \begin{array}{l}\text { Eu faço coisas sem } \\ \text { pensar. }\end{array} \\ \begin{array}{l}\text { Imake-up my mind } \\ \text { quickly. }\end{array} & \begin{array}{l}\text { Eu tomo decisões } \\ \text { rapidamente. }\end{array} & \text { Imake decisions quickly. } & \text { Eu tomo decisões } \\ \text { rapidamente. }\end{array}$

$4 \quad$ I am happy-go-lucky.

Eu sou despreocupado

I don't worry (I believe in (confio na sorte, "desen- luck, "carefree"). canado").

$\begin{array}{llll}\text { I don't "pay attention". } & \text { Eu não presto atenção. } & \text { I don't pay attention. } & \text { Eu não presto atenção. } \\ \text { I have "racing" thoughts. } & \begin{array}{l}\text { Eu tenho pensamentos } \\ \text { que se atropelam. }\end{array} & \begin{array}{l}\text { I have thoughts that get } \\ \text { in the way. }\end{array} & \begin{array}{l}\text { Eu tenho pensamentos } \\ \text { que se atropelam. }\end{array}\end{array}$

Eu planejo viagens com bastante antecedência.

Eu tenho autocontrole. I concentrate easily.

Eu me concentro facilmente.

Eu economizo (poupo) regularmente.

I "squirm" at plays or lectures. I plan for job security. thinking. complex problems.

I change jobs.

17 when solving thought problems. cuidado.

Eu faço planos para me manter no emprego (eu cuido para não perder meu emprego).

\section{Eu falo coisas sem} pensar.

Eu gosto de pensar em problemas complexos.

Eu fico me contorcendo na cadeira em peças de teatro ou palestras.

petrestless during plays and lectures.

I think about things carefully.

I make plans to keep my job (I take care to not lose my job).

I say things without thinking.

Ilike to think about complex problems.

Eu troco de emprego.

Eu ajo por impulso.

Eu fico entediado com facilidade quando estou resolvendo problemas mentalmente.

Eu ajo no "calor" do momento.

I get easily bored when I'm solving problems mentally.

I act in the "heat" of the moment.
I plan trips well in advance.

I have self control.

Eu planejo viagens com bastante antecedência.

Eu tenho autocontrole.

Eu me concentro facilmente.

Eu economizo (poupo) regularmente.

Eu fico me contorcendo na cadeira em peças de teatro ou palestras.

Eu penso nas coisas com cuidado.

Eu faço planos para me manter no emprego (eu cuido para não perder meu emprego).

Eu falo coisas sem pensar.

Eu gosto de pensar em problemas complexos.

Eu troco de emprego.

Eu ajo por impulso.

Eu fico entediado com resolvendo problemas mentalmente.

Eu ajo no "calor" do momento.
Eu sou despreocupado (confio na sorte, "desencanado").

Eu não presto atenção.

A tradução manteve integralmente os componentes literal, semântico e idiomático da versão em inglês.

A expressão presente no idioma inglês "make up my mind" tem 0 sentido de assumir uma ideia, uma determinação mental. Assim, a tradução manteve integralmente o componente literal e semântico da versão em inglês.

A conotação de despreocupado à qual 0 item se refere envolve a adoção de comportamentos sem pensar em suas consequências. Por isso, optou-se por acrescentar entre parênteses a expressão "confio na sorte" e o termo "desencanado". Assim, a tradução manteve integralmente os componentes literal e semântico da versão em inglês.

A tradução manteve integralmente os componentes literal, semântico e idiomático da versão em inglês.

A expressão "racing thoughts" pode parecer em princípio algo equivalente a pensamentos rápidos. No entanto, seu uso se refere a um padrão de pensamentos acelerados que saem do controle, como ocorre nos quadros maníacos. Assim, optamos pelas equivalências literal e semântica da tradução inicial.

A tradução manteve a equivalência literal, semântica e idiomática.

A tradução manteve a equivalência literal, semântica e idiomática.

A tradução manteve integralmente os componentes semânticos e literais da versão em inglês.

A tradução manteve integralmente os componentes semânticos e literais da versão em inglês.

Na BIS-11, os itens 11 e 28 são semelhantes. No entanto, 0 item 11 pertence à subescala motora e, por isso, optou-se pela tradução em que o significado reporta à atividade de se contorcer durante atividades como peças de teatro ou palestras.

A tradução manteve a equivalência literal, semântica e idiomática.

A tradução manteve a equivalência idiomática e semântica.

A tradução manteve integralmente os componentes literal, semântico e idiomático da versão em inglês.

A tradução manteve integralmente os componentes literal, semântico e idiomático da versão em inglês.

A tradução manteve integralmente os componentes literal, semântico e idiomático da versão em inglês.

A tradução manteve integralmente os componentes literal, semântico e idiomático da versão em inglês. facilidade quando estou

A tradução manteve integralmente os componentes literal, semântico e idiomático da versão em inglês.
A tradução manteve integralmente os componentes literal, semântico e idiomático da versão em inglês. 
Anexo 1. Procedimentos do processo de tradução do instrumento BIS-11

\begin{tabular}{|c|c|c|c|c|c|}
\hline & \multicolumn{4}{|c|}{ Itens } & \multirow{2}{*}{$\begin{array}{l}\text { Síntese da análise comparativa da tradução e retrotradução } \\
\text { (etapa 3) }\end{array}$} \\
\hline & Versão original & $\begin{array}{l}\text { Versão traduzida } \\
\text { (etapa 1) }\end{array}$ & $\begin{array}{l}\text { Versão retrotradução } \\
\text { (etapa 2) }\end{array}$ & $\begin{array}{l}\text { Versão final } \\
\text { (etapa 3) }\end{array}$ & \\
\hline 20 & I am a steady thinker. & $\begin{array}{l}\text { Eu mantenho a linha de } \\
\text { raciocínio ("não perco } 0 \\
\text { fio da meada"). }\end{array}$ & $\begin{array}{l}\text { I maintain the line of } \\
\text { reasoning ("I don't lose } \\
\text { the thread"). }\end{array}$ & $\begin{array}{l}\text { Eu mantenho a linha de } \\
\text { raciocínio ("não perco } 0 \\
\text { fio da meada"). }\end{array}$ & $\begin{array}{l}\text { Optou-se pelo sentido de sustentação da linha de raciocínio, } \\
\text { uma vez que reflete o sentido de estabilidade do pensamento. }\end{array}$ \\
\hline 21 & I change residences. & $\begin{array}{l}\text { Eu troco de casa (resi- } \\
\text { dência). }\end{array}$ & I move (residence). & $\begin{array}{l}\text { Eu troco de casa (resi- } \\
\text { dência). }\end{array}$ & $\begin{array}{l}\text { A tradução manteve integralmente os componentes literal, } \\
\text { semântico e idiomático da versão em inglês. }\end{array}$ \\
\hline 22 & I buy things on impulse. & $\begin{array}{l}\text { Eu compro coisas por } \\
\text { impulso. }\end{array}$ & I buy things on impulse. & $\begin{array}{l}\text { Eu compro coisas por } \\
\text { impulso. }\end{array}$ & $\begin{array}{l}\text { A tradução manteve integralmente os componentes semânti- } \\
\text { cos e literais da versão em inglês. }\end{array}$ \\
\hline 23 & $\begin{array}{l}\text { I can only think about } \\
\text { one thing at a time. }\end{array}$ & $\begin{array}{l}\text { Eu só consigo pensar } \\
\text { em um problema de } \\
\text { cada vez. }\end{array}$ & $\begin{array}{l}\text { I am able to think about } \\
\text { only one problem at } \\
\text { a time. }\end{array}$ & $\begin{array}{l}\text { Eu só consigo pensar em } \\
\text { uma coisa de cada vez. }\end{array}$ & $\begin{array}{l}\text { Após a retrotradução, optou-se pela substituição de "proble- } \\
\text { ma" por "coisa". }\end{array}$ \\
\hline 24 & I change hobbies. & $\begin{array}{l}\text { Eu troco de interesses e } \\
\text { passatempos ("hobby"). }\end{array}$ & $\begin{array}{l}\text { I change interests and } \\
\text { pastimes ("hobby"). }\end{array}$ & $\begin{array}{l}\text { Eu troco de interesses e } \\
\text { passatempos ("hobby"). }\end{array}$ & $\begin{array}{l}\text { Adotamos dois equivalentes semânticos do termo "hobbies" } \\
\text { para facilitar a compreensão do item em diferentes contextos } \\
\text { educacionais e por diferentes níveis sociais. }\end{array}$ \\
\hline 25 & $\begin{array}{l}\text { I spend or charge more } \\
\text { than I earn. }\end{array}$ & $\begin{array}{l}\text { Eu gasto ou compro a } \\
\text { prestação mais do que } \\
\text { ganho. }\end{array}$ & $\begin{array}{l}\text { I spend or buy on credit } \\
\text { more than I make. }\end{array}$ & $\begin{array}{l}\text { Eu gasto ou compro a } \\
\text { prestação mais do que } \\
\text { ganho. }\end{array}$ & A tradução manteve a equivalência semântica. \\
\hline 26 & $\begin{array}{l}\text { I often have extraneous } \\
\text { thoughts when thinking. }\end{array}$ & $\begin{array}{l}\text { Enquanto estou pen- } \\
\text { sando em uma coisa, é } \\
\text { comum que outras ideias } \\
\text { me venham à cabeça ou } \\
\text { ao mesmo tempo. }\end{array}$ & $\begin{array}{l}\text { When I am thinking } \\
\text { about one thing, it is } \\
\text { common that other ideas } \\
\text { come into my head or at } \\
\text { the same time. }\end{array}$ & $\begin{array}{l}\text { Enquanto estou pen- } \\
\text { sando em uma coisa, é } \\
\text { comum que outras ideias } \\
\text { me venham à cabeça ou } \\
\text { ao mesmo tempo. }\end{array}$ & A tradução manteve a equivalência semântica. \\
\hline 27 & $\begin{array}{l}\text { I am more interested } \\
\text { in the present than the } \\
\text { future. }\end{array}$ & $\begin{array}{l}\text { Eu tenho mais interesse } \\
\text { no presente do que no } \\
\text { futuro. }\end{array}$ & $\begin{array}{l}\text { I am more interested } \\
\text { in the present than the } \\
\text { future. }\end{array}$ & $\begin{array}{l}\text { Eu tenho mais interesse } \\
\text { no presente do que no } \\
\text { futuro. }\end{array}$ & $\begin{array}{l}\text { A tradução manteve integralmente os componentes literal, } \\
\text { semântico e idiomático da versão em inglês. }\end{array}$ \\
\hline 28 & $\begin{array}{l}\text { I am restless at the } \\
\text { theater or lectures. }\end{array}$ & $\begin{array}{l}\text { Eu me sinto inquieto em } \\
\text { palestras ou aulas. }\end{array}$ & $\begin{array}{l}\text { I feel restless during } \\
\text { lectures and classes. }\end{array}$ & $\begin{array}{l}\text { Eu me sinto inquieto em } \\
\text { palestras ou aulas. }\end{array}$ & $\begin{array}{l}\text { Na BIS-11, os itens } 11 \text { e } 28 \text { são muito parecidos. No entanto, } 0 \\
\text { item } 28 \text { pertence à subescala atencional e, por isso, optou-se } \\
\text { pela tradução em que o significado reporta ao sentimento } \\
\text { de inquietude sem implicar manifestações motoras durante } \\
\text { atividades como peças de teatro ou palestras. }\end{array}$ \\
\hline 29 & I like puzzles. & $\begin{array}{l}\text { Eu gosto de jogose } \\
\text { desafios mentais. }\end{array}$ & $\begin{array}{l}\text { I like mentally challen- } \\
\text { ging games. }\end{array}$ & $\begin{array}{l}\text { Eu gosto de jogose } \\
\text { desafios mentais. }\end{array}$ & $\begin{array}{l}\text { A tradução manteve integralmente os componentes literal, } \\
\text { semântico e idiomático da versão em inglês. }\end{array}$ \\
\hline 30 & I am future oriented. & $\begin{array}{l}\text { Eu me planejo para } 0 \\
\text { futuro. }\end{array}$ & I plan for the future. & $\begin{array}{l}\text { Eu me preparo para } 0 \\
\text { futuro. }\end{array}$ & $\begin{array}{l}\text { Embora a tradução inicial tenha mantido a equivalência se- } \\
\text { mântica, considerou-se que o termo "me preparo" contempla } \\
\text { o sentido que o item busca: orientar as ações em direção ao } \\
\text { futuro. }\end{array}$ \\
\hline
\end{tabular}

b) Análise da consistência das respostas da amostra 1 aos itens das versões originais e em português da escala:

Com base nas respostas da amostra bilíngue 1, foram obtidos 30 coeficientes de correlações de Spearman a partir da associação entre os itens originais e os itens traduzidos. Onze itens (cerca de 37\% do total) evidenciaram índices fortes de correlação $(0,80=<$ rho $<0,91)$, dezesseis itens (cerca de $33 \%$ do total) apresentaram correlações moderadas e significativas $(0,40=<$ rho $<0,80)$ e apenas três itens $(4,20$ e 26) apresentaram correlações não significativas $(p>0,05)$.

Os resultados da análise item-total indicam que, dentre os três itens (4, 20 e 26) que apresentaram correlações não significativas entre as respostas às versões original e traduzida, o item 26 foi o único que apresentou correlação itemtotal moderada (em torno de 0,40 ) com os demais itens que compõem a subescala atencional. Os itens 4 e 20 apresentaram correlações muito fracas com o escore total da versão em inglês $(0,05)$ e com o escore total da versão em português $(0,32$ e 0,01, respectivamente). Os demais itens do instrumento apresentaram correlação item-total moderada-alta nas versões em português e inglês. A hipótese sugerida para explicar esse fato é que os itens 4, 20 e 26 contêm gírias na versão americana (item 4 - "happy-go-lucky"; item 20 - "steady thinker"; item 26 - "extraneous thoughts") e que não apresentavam uma expressão equivalente no idioma português. Nesse sentido, para se obter uma compreensão aprimorada das dificuldades em relação aos itens 4, 20 e 26, que não apresentaram índices não significativos de correlação, optou-se por realizar nova aplicação das duas versões da escala em uma amostra com proficiência em inglês e cujos participantes residissem nos Estados Unidos por tempo superior a cinco anos.

De um total de 30 correlações de Spearman efetuadas entre os itens originais e os traduzidos, a maioria das correlações foram moderadas e significativas $(0,30<$ rho $<0,80)$. É interes- 
sante notar que os itens 20 e 26, que apresentaram correlações não significativas nas análises realizadas a partir das respostas da amostra bilíngue 1 , foram significativos na amostra bilíngue 2 (0,51 para o item 4 - "happy-go-lucky"; 0,87 para o item 20 - "steady thinker" e 0,70 para o item 26 - "extraneous thoughts"). Essa constatação é uma evidência de que tais itens apresentam gírias idiomáticas, o que pode ter contribuído para o não entendimento dos participantes que possuíam um domínio avançado do idioma inglês. Diante da análise das respostas, pode-se concluir que as baixas correlações obtidas no estudo anterior relativas aos itens 4, 20 e 26 se devem à dificuldade de traduzir as expressões assinaladas acima para o idioma português. Sabe-se que determinadas palavras de um idioma podem não possuir uma tradução exata para outro idioma. Além disso, o escore total da versão americana foi correlacionado ao escore total da versão brasileira a partir dos dados obtidos nas duas amostras bilíngues. Encontrou-se um escore significativo equivalente $\mathrm{r}=0,93$ e $r=0,91(p<0,001)$. Os índices de correlação entre os escores parciais das duas versões variaram entre 0,80 e 0,91 ( $p<0,001)$.

c) Análise quantitativa das equivalências literal, idiomática e conceitual dos itens das duas versões:

A análise da frequência relativa das respostas dos avaliadores quanto à pertinência da tradução do item 20 em relação aos critérios de equivalência idiomática e semântica indicou que a maioria dos avaliadores (acima de 70\%) considerou pertinente a tradução proposta. Em relação ao item 24, o grau de concordância para os critérios de equivalência idiomática e semântica foi de 100\% e igual a $85 \%$ para a equivalência literal, o que indica que a maioria dos avaliadores concorda com a pertinência da tradução. Por fim, a análise da frequência relativa das respostas dos avaliadores para o item 26 indicou que $65 \%$ dos avaliadores consideraram equivalente a tradução para a língua portuguesa quanto aos critérios de equivalência idiomática e semântica. É interessante notar que a avaliação da equivalência literal da tradução apresentou baixos índices de concordância, sendo igual a 20\% para o item 20 e a 0\% para o item 26. Essa informação sugere que tais itens são compostos por expressões no idioma inglês ("steady thinker" e "extraneous thoughts") que não possuem uma equivalência direta na língua portuguesa, o que justifica os baixos coeficientes de correlação entre as respostas da versão original e na língua portuguesa na amostra bilíngue 1 .

\section{DISCUSSÃO}

Conforme destacado por Mattos et al. ${ }^{11}$, a sistemática das traduções de instrumentos para línguas e culturas diferentes tem sido bastante debatida. O processo deve ser minucioso, envolvendo não apenas a tradução por especialistas, mas a análise da equivalência conceitual/semântica, a análise específica de itens e a consistência entre a versão origi- nal e a versão adaptada. É de crucial importância a adoção de cuidados linguísticos, na medida em que determinados termos podem ter diferentes abrangências, especificidades e conotações, inerentes a cada idioma ou cultura. Assim, a equivalência literal na tradução de um instrumento pode ser insuficiente para a manutenção de seu objetivo quando usado em uma nova cultura. Tal fato pode ser observado no presente estudo com relação aos itens 4, 20 e 26 da escala. Esses itens, embora não tenham apresentado boas propriedades psicométricas relativas à tradução literal, mostraramse equivalentes em termos idiomáticos e semânticos quando analisados por juízes com elevada proficiência em inglês e português. A importância de se buscar equivalência entre a versão em língua estrangeira e o português tem sido cada vez mais reconhecida, crescendo o número de estudos que, em diferentes campos, buscam o desenvolvimento de instrumentos considerando essa preocupação ${ }^{11-15}$.

O presente trabalho apresenta uma adaptação brasileira de um dos instrumentos mais utilizados na literatura para avaliação da impulsividade. A BIS-11 tem se mostrado útil na caracterização dos diferentes tipos de impulsividade medidos pelo modelo de Barratt e tem sido traduzida para diversos idiomas desde sua primeira publicação há 50 anos $^{16}$. Ela tem se mostrado útil na identificação de diferentes padrões de impulsividade em diversas patologias, como o transtorno do déficit de atenção e hiperatividade ${ }^{3}$, o transtorno afetivo bipolar ${ }^{17}$, a esquizofrenia ${ }^{18}$, o transtorno por uso de substância ${ }^{19}$, o jogo patológico ${ }^{3}$, entre outras. Além disso, tem sido bastante útil em estudos em neurobiologia da impulsividade ${ }^{20}$, bem como em estudos sobre outros construtos neuropsicológicos relacionados à impulsividade, tais como funções executivas ${ }^{21}$, atençãa ${ }^{22}$ e sensibilidade ao reforço ${ }^{23}$. Deve ser salientado ainda que o modelo proposto por Barratt apresenta importante relação com outros modelos em neurociências sobre impulsividade $\mathrm{e}^{2-3}$ e sobre aspectos anatomofuncionais dos circuitos pré-frontais, como no modelo proposto por Fuster ${ }^{24}$. A despeito das diferenças culturais relacionadas a conceitos relativos aos diferentes tipos de impulsividade, o modelo de Barratt e a BIS-11 têm sido adaptados com sucesso para diferentes culturas, evidenciando sua validade transcultural. Dada a importância do modelo proposto por Barratt e da BIS-11 para estudos sobre a impulsividade, considera-se que o desenvolvimento de uma versão brasileira da escala é de fundamental importância para a prática clínica e para a realização de estudos sobre o tema.

Deve ser salientado que o presente trabalho apresenta a adaptação da versão 11 da Barratt Impulsiveness Scale, sendo necessária a realização de estudos sobre validade de critério e de construto, sobre a estrutura fatorial da escala adaptada e também sobre o desempenho de um grupo normativo de brasileiros de diferentes estados para avaliação de possíveis diferenças regionais no desempenho da escala. Tais estudos, em andamento, possibilitarão o uso adequado da escala na clínica e na pesquisa em nosso contexto. 


\section{AGRADECIMENTOS}

Este projeto recebeu apoio financeiro da FAPEMIG (SHAAPQ-00741-09); Secretaria de Estado Ciência, Tecnologia e Ensino Superior (MG) - Emenda Parlamentar 654/2008; Deputado Lafaiete Andrada.

\section{REFERÊNCIAS}

1. Moeller FG, Barratt ES, Dougherty DM, Schmitz JM, Swann AC. Psychiatric aspects of impulsivity. Am J Psychiatry. 2001;158:1783-93.

2. Fuentes D, Tavares H, Artes R, Gorenstein C. Self-reported and neuropsychological measures of impulsivity in pathological gambling. J Int Neuropsychol Soc. 2006;12(6):907-12.

3. Malloy-Diniz LF, Fuentes D, Leite WB, Correa H, Bechara A. Impulsive behavior in adults with attention deficit/hyperactivity disorder: characterization of attentional, motor and cognitive impulsiveness. J Int Neuropsychol Soc. 2007;13(4):693-8.

4. Malloy-Diniz L, Neves F, Abrantes S, Fuentes D, Correa H. Suicide behavior and neuropsychological assessment of type I bipolar patients. J Affect Disord. 2009;112:231-6.

5. Salgado JV, Malloy-Diniz LF, Abrantes SSC, Campos VR, Fuentes D, Bechara A, et al. Neuropsychological assessment of impulsive behavior in short-term abstinent alcohol-dependent subjects. Rev Bras Psiquiatr. 2009;31:4-9.

6. Tavares H, Lobo DSS, Fuentes D, Black DW. Compras compulsivas: uma revisão e um relato de caso. Rev Bras Psiquiatr. 2008;30:516-23.

7. Araujo MM, Malloy-Diniz LF, Rocha FL. Impulsividade e acidentes de trânsito: artigo de revisão. Rev Psiq Clin. 2009;36:55-64.

8. Patton JH, Stanford MS, Barratt ES. Factor structure of the Barratt Impulsiveness Scale. J Clin Psychol. 1995:51(6):768-74.

9. Diemen L, Szobot CM, Kessler F, Pechansky F. Adaptation and construct validation of the Barratt Impulsiviness Scale (BIS-11) to Brazilian Portuguese for use in adolescents. Rev Bras Psiquiatr. 2007;29(2):153-6.

10. Barratt, ES. Anxiety and impulsiveness related to psychomotor efficiency. Percept Mot Skills. 1959;9(2):191-8.
11. Mattos P, Serra-Pinheiro MA, Rohde LA, Pinto D. Apresentação de uma versão em português para uso no Brasil do instrumento MTA-SNAP-IV de avaliação de sintomas de transtorno do déficit de atenção/hiperatividade e sintomas de transtorno desafiador e de oposição. Revista de Psiquiatria do Rio Grande do Sul. 2006;28(3):290-7.

12. Souza I, Serra MA, Mattos P, Franco VA. Comorbidade em crianças e adolescentes com transtorno do déficit de atenção. Arq Neuropsiquiatr. 2001;59(2-B):401-6.

13. DuPaul GJ, Power TJ, Anastopoulos AD, Reid R. ADHD Rating Scales-IV: checklists, norms and clinical interpretation. New York: Guilford Press; 1998.

14. Santos CB, Carvalho SCA, Silva MFG, Fuentes D, Santana PA, Furlan AB, et al. Cross-cultural adaptation of the Innsbruck health dimensions questionnaire for neurosurgical patients (IHD-NS). Arq Neuropsiquiatr. 2008:66:698-701.

15. Hu LW, Gorenstein C, Fuentes D. Portuguese version of Corah's Dental Anxiety Scale: transcultural adaptation and reliability analysis. Depress Anxiety. 2007;24:467-71.

16. Stanford MS, Mathias CW, Dougherty DM, Lake, SL, Anderson NE, Patton JH. Fifty years of the Barratt Impulsiveness Scale: an update and review. Pers Individ Dif. 2009;47(5):385-95.

17. Swann AC, Pazzaglia P, Nicholls A, Dougherty DM, Moeller FG. Impulsivity and phase of illness in bipolar disorder. J Affect Dis. 2003;73:105-11

18. Enticott PG, Ogloff JRP, Bradshaw, JL, Fitzgerald PB. Cognitive inhibitory control and selfreported impulsivity among violent offenders with schizophrenia. J Clin Exp Neuropsychol. 2008;30:1-6.

19. Lejuez (W, Bornovalova, MA, Reynolds EK, Daughters SB, Curtin JJ. Risk factors in the relationship between gender and crack/cocaine. Exp Clin Psychopharmacol. 2007;15:165-75

20. Paaver M, Nordquist N, Parik J, Harro M, Oreland L, Harro J. Platelet MA0 activity and the 5-HTT gene promoter polymorphism are associated with impulsivity and cognitive style in visual information processing. Psychopharmacology. 2007;194:545-54

21. Cheung AM, Mitsis EM, Halperin JM. The relationship of behavioral inhibition to executive functions in young adults. J Clin Exp Neuropsychol. 2004;26:393-404

22. Levine LE, Waite BM Bowman LL. Electronic media use, reading, and academic distractibility in college youth. Cyberpsychology Behav. 2007;10:560-6.

23. Martin LE, Potts GF. Reward sensitivity in impulsivity. Cognitive Neurosci Neuropsychol. 2004;15:1519-22.

24. Fuster JM. The prefrontal cortex. Amsterdam: Academic Press/Elsevier; 2008. 